\title{
INTERAÇÃO COBRE, MOLIBDÊNIO E ENXOFRE EM RUMINANTES
}

\author{
COPPER, MOLYBDENUM AND SULPHUR INTERACTION IN RUMINANT NUTRITION
}

\author{
Eduardo Fabián Aragón Vásquez ${ }^{1}$ Alexandra del Pilar Naranjo Herrera ${ }^{2}$ Genario Sobreira Santiago ${ }^{3}$
}

\section{- REVISÃo BIBLIOGRÁFICA -}

RESUMO

\begin{abstract}
O cobre $(\mathrm{Cu})$ é um componente essencial a varias funções no metabolismo animal. A deficiência de $\mathrm{Cu}$ em ruminantes é endêmica em varias regiões ao redor do mundo, especialmente quando as pastagens são altas em molibdênio. $\mathrm{O} C \mathrm{Cu}, \mathrm{Mo}$ e enxofre podem se combinar no rúmen para formar complexos triplos não absorvíveis denominados cupro-tiomolibdatos (CuTMs). O efeito fisiológico importante dos Cu-TMs está na restrição da disponibilidade de Cu para a síntese de ceruloplasmina. Os ruminantes, especialmente ovinos, são muito mais susceptíveis ao inbalanço Cu:Mo que animais não ruminantes pela relação entre as bactérias rumenais com a geração de sulfeto. Os efeitos de um determinado nível de $\mathrm{Cu}$ dietéticos são altamente influenciados pelo índice deste cobre com o molibdênio e enxofre dietéticos.
\end{abstract}

Palavras-chave: cobre, molibdênio, enxofre, tiomolibdatos.

\section{SUMMARY}

Copper is an essential component of several functions in animal metabolism. Ruminat $\mathrm{Cu}$ deficiency is endemic in regions throughout the world, specially when the pasture are rich in molybdenum. $\mathrm{Cu}, \mathrm{Mo}$ and sulphur could combine in the rumen to form an unabsorbable triple complex nominated copper thiomolybdate $(\mathrm{Cu}$-TMs). The important physiological effects of $\mathrm{Cu}$-TMs is to restrict the availability of $\mathrm{Cu}$ for ceruloplasmin synthesis. Ruminant animals, especially sheep, are much more susceptible to Cu:Mo imbalance than are nonruminant animals because of the sulfide generating bacteria in the rumen. The effects of a particular level of dietary $C u$ are also greatly influenced by the ratio of this copper to dietary Mo and sulphate.

Key words: copper, molybdenum, sulphur, thiomolybdates.

\section{INTRODUÇÃO}

O papel e importância do cobre $(\mathrm{Cu})$ no metabolismo animal são bem conhecidos. A deficiência e toxicidade do $\mathrm{Cu}$ em ruminantes ocorrem freqüentemente em muitas partes do mundo. A mais importante deficiência de origem mineral depois do fósforo, talvez seja a de $\mathrm{Cu}$ (MILTIMORE \& MASON, 1971). O desenvolvimento da deficiência de $\mathrm{Cu}$ depende tanto da concentração de $\mathrm{Cu}$ na dieta como das concentrações dos antagonistas que interferem com a absorção e a subseqüente utilização para os processos metabólicos (GOONERATNE $\boldsymbol{e t}$ al., 1989).

A deficiência "simples" causada pela ingestão de quantidades pequenas de $\mathrm{Cu}$ dietético é freqüentemente responsável pelo desenvolvimento de desordens clínicas como: anormalidades na pigmentação e queratinização da lã e pêlo, anemia, anormalidades esqueléticas e ataxia enzoótica (GOONERATNE $\boldsymbol{e t}$ al., 1989). Embora seja comum

\footnotetext{
${ }^{1}$ Médico Veterinário e Zootecnista, Mestre em Zootecnia, Doutorando em Ciência Animal. EV/Universidade Federal de Minas Gerais (UFMG), Escola de Veterinária da UFMG, Departamento de Zootecnia. Av. Antônio Carlos 6627, CP 567, 30161-970 Belo Horizonte, MG. E-mail: eduardoaragon@yahoo.com. Autor para correspondência.

${ }^{2}$ Médico Veterinário e Zootecnista, Mestre em Zootecnia EV/UFMG.

${ }^{3}$ Médico Veterinário, Professor Adjunto de Bioquímica Veterinária da Faculdade de Veterinária da Universidade Estadual do Ceará, Mestre em Zootecnia, Doutorando em Ciência Animal EV/UFMG. 
observar-se deficiência de $\mathrm{Cu}$ em ruminantes a pasto com concentrações, aparentemente adequadas de $\mathrm{Cu}$ (DICK et al., 1975); esta deficiência "induzida" ou "condicionada" é causada pela presença de antagonistas que reduzem a disponibilidade de $\mathrm{Cu}$. Molibdênio (Mo) e enxofre (S) têm sido identificados como os antagonistas do $\mathrm{Cu}$ de maior importância (HUMPHRIES et al., 1983). McDOWELL et al. (1988) categorizaram as deficiências de cobre em três grupos: alimentos com níveis altos de Mo, baixo teor de $\mathrm{Cu}$ porem quantidades significativas de $\mathrm{Mo}$ (<2:1), e conteúdo normal de $\mathrm{Cu}$ e baixo de $\mathrm{Mo}$, com altos níveis de proteína solúvel. A ultima situação é o resultado do elevado consumo de proteína solúvel encontrada nos pastos novos, que aumenta o conteúdo de sulfito no rúmen, resultando na formação de sulfito de cobre que não é utilizável. Este artigo tem como objetivo estudar a interação $\mathrm{Cu}$ Mo -S e a formação de tiomolobdatos em ruminantes.

\section{COBRE - MOLIBDÊNIO - ENXOFRE}

O conteúdo de cobre nas dietas para ruminantes geralmente varia entre 4 a $10 \mathrm{mg} / \mathrm{kg}$ de $\mathrm{MS}$, sendo bem conhecido o risco de toxicidade por $\mathrm{Cu}$ nesta classe de animais (GOONERATNE $\boldsymbol{e t}$ al., 1989). A excreção urinária de $\mathrm{Cu}$ tem sido considerada como de menor importância comparada a biliar, podendo constituir $25 \%$ do total das perdas e sendo maior em animais jovens que em velhos. A adição de $\mathrm{Mo}$ e $\mathrm{S}$ a dietas com nível adequado de $\mathrm{Cu}$, aumenta a excreção urinária desse último (SUTTLE, 1991).

A concentração de molibdênio no pasto, freqüentemente, encontra-se entre 0,5 a $5 \mathrm{mg} / \mathrm{kg}$ de MS (GOONERATNE et al., 1989), variando mais que o $\mathrm{Cu}$. Ao contrário do $\mathrm{Cu}$, a concentração de $\mathrm{Mo}$ incrementa quando o $\mathrm{pH}$ do solo aumenta (GALBRAITH $\boldsymbol{e t} \boldsymbol{a l}$., 1997). LADEFOGED \& STÜRUP (1995) manifestam que ao adicionar-se Mo em excesso à silagem para vacas observam-se alterações na pele e diarréia justamente quando as concentrações séricas de $\mathrm{Cu}$ e ceruloplasmina permanecerem altas.

O conteúdo de $\mathrm{S}$ no pasto depende grandemente de sua concentração nas proteínas e dificilmente excede $3 \mathrm{~g} / \mathrm{kg}$ de MS. A metionina e cisteína geralmente contêm mais de $90 \%$ do enxofre orgânico da forragem (GOONERATNE et al., 1989). Dos três componentes da interação, o $\mathrm{S}$ apresenta maior variação por causa das vias metabólicas alternativas do rúmen. A saída de $\mathrm{S}$ do rúmen é principalmente por absorção como sulfeto $\left(\mathrm{S}^{2-}\right)$, porém também por excreção na forma de enxofre protéico ou proteína microbiana. Só o enxofre protéico degradado e o $\mathrm{S}$ inorgânico proveniente da dieta ou saliva são disponíveis para interagir com Mo e $\mathrm{Cu}$ no rúmen. Dietas ricas em enxofre, porém pobres em Mo incrementam a concentração ruminal de sulfeto e diminuem a disponibilidade de $\mathrm{Cu}$ em ovelhas (SUTTLE, 1991). A suplementação com sulfato pode contribuir para aumentar a excreção urinária de $\mathrm{Cu}$ (<1mg Cu/dia) (MASON et al., 1982).

TOKARNIA et al. (1999) apresentaram uma revisão dos estudos realizados no Brasil durante o período de 1987 a 1998, mostrando que grandes partes dos solos brasileiras produziram forrageiras com baixos níveis de $\mathrm{Cu}$. Já para Mo, existiu grande variação, embora haja pouca investigação deste mineral, se conhece que na sub-região Nhecolândia (parte central) do Pantanal mato-grossense existem níveis normais a baixos tanto no solo como nos hepatócitos de bovinos da região (POTT et al., 1989), porém nas regiões Centro-Oeste e Norte, demonstrou-se que nas pastagens existem teores elevados de molibdênio, e principalmente de enxofre (MORAES et al., 1994). Pesquisas realizadas na ilha de Marajó (Pará), com bovinos e búfalos, CARDOSO et al. (1997) mostraram que a concentração media de $\mathrm{Cu}$ e Mo nas forragens foi menor que os valores críticos e tóxicos respectivamente. A relação $\mathrm{Cu}: \mathrm{Mo}(1,2: 1)$ também foi menor que o mínimo recomendado $(2: 1)$, indicando valores críticos para cobre.

\section{OS TIOMOLIBDATOS}

Os efeitos adversos de aumentar o Mo e S dietéticos sobre a utilização de $\mathrm{Cu}$ pelos ruminantes têm sido atribuídos à formação de tiomolibdatos (TMs ou $\mathrm{MoS}_{4}{ }^{2-}$ ) no ambiente ruminal rico em sulfeto (DICK $\boldsymbol{e t}$ al., 1975; SUTTLE, 1974). Os efeitos sistêmicos dos TMs envolvem principalmente a inibição do metabolismo do $\mathrm{Cu}$ (MILTIMORE \& MASON, 1971). As descobertas mostram que os tetratiomolibdatos (TM4) parecem ser dominantes no rúmen com aportes moderadamente altos de Mo e S. Segundo SUTTLE (1991), as porcentagens com que participam os TMs são: $41 \%$ como tetratiomolibdato $\left(\mathrm{TM}_{4}\right.$ ou $\left.\mathrm{MoS}_{4}{ }^{2-}\right), 34 \%$ como tritiomolibdato $\left(\mathrm{TM}_{3}\right.$ ou $\left.\mathrm{MoS}_{3}{ }^{2-}\right)$, e monotiomolibdato $\left(\mathrm{TM}_{1}\right.$ ou $\left.\mathrm{MoO}_{3} \mathrm{~S}^{2-}\right)$ e ditiomolibdato $\left(\mathrm{TM}_{2}\right.$ ou $\left.\mathrm{MoO}_{2} \mathrm{~S}^{2-}\right)$ em menores quantidades. A reação para a formação de mono, di, tri e tetratiomolibdato sugerida por SUTTLE (1974) é mostrada a seguir:

$\mathrm{MoO}_{4}{ }^{2-}+\mathrm{S}^{2-} \rightarrow \mathrm{MoO}_{3} \mathrm{~S}^{2-}+\mathrm{S}^{2-} \rightarrow \mathrm{MoO}^{2-} \mathrm{S}^{2-}+$ $\mathrm{S}^{2-} \rightarrow \mathrm{MoOS}_{3}{ }^{2-}+\mathrm{S}^{2-} \rightarrow \mathrm{MoS}_{4}{ }^{2-}$

Os TMs ligam-se com o $\mathrm{Cu}$, no rúmen, para formar cupro-tiomolibdatos (Cu-TMs) que são 
insolúveis e indisponíveis para a absorção (MASON, 1990). Os TMs ligam-se aos sólidos do rúmen e, quando a concentração de TMs é alta, também com a fase líquida do rúmen. Os excessos de TMs, quando absorvidos, passam ao sangue e produzem distúrbios sistêmicos no metabolismo do $\mathrm{Cu}$; estas características fazem com que a concentração plasmática de cobre aumente. Muito deste $\mathrm{Cu}$ está ligado a proteínas, sendo insolúvel em ácido tricloroacético (ATC) e aparentemente não disponível para a entrada nos tecidos (PRICE $\boldsymbol{e t}$ al., 1987). A formação de $\mathrm{Cu}$-TMs provoca diminuição na concentração tissular de $\mathrm{Cu}$, eleva o $\mathrm{Cu}$ plasmático, e surgem os sinais de deficiência de $\mathrm{Cu}$ (MASON et al., 1982). Os CuTMs associam-se às proteínas de alto peso molecular, principalmente albumina e formam um complexo insolúvel e, assim, reduz-se a absorção cúprica (SUTTLE, 1991). Este complexo com a albumina é tão forte que é precipitado sobre acidificação, o que explica, a princípio, o descobrimento da fração insolúvel ATC no plasma de carneiros expostos a Mo e S (GALBRAITH et al., 1997). O complexo CuTM-albumina apresenta um tempo de vida média maior que $\mathrm{Cu}$-albumina em vacas. $\mathrm{O}$ efeito fisiológico importante da formação do complexo $\mathrm{Cu}$-TMalbumina na corrente sangüínea relaciona-se à restrição da disponibilidade de $\mathrm{Cu}$ para síntese de ceruloplasmina (cobre mono amino oxidase, $\mathrm{Cu}-\mathrm{MAO}$ ), fração alfa-2 globulina do sangue, em que cerca de 95\% do $\mathrm{Cu}$ sérico encontra-se ligado (GOONERATNE et $\boldsymbol{a l}$., 1989). A ceruloplasmina contém três oligosacarídeos ligados por asparagina e oito sítios que ligam o $\mathrm{Cu}^{+}$ou $\mathrm{Cu}^{2+}$. Trata-se de uma proteína (enzima) que funciona como Ferroxidase, convertendo o $\mathrm{Fe}^{2+}$ em $\mathrm{Fe}^{3+}$. Ajuda também na manutenção da homeostase do $\mathrm{Cu}^{2+}$ e serve no transporte de $\mathrm{Cu}^{2+}$ (SMITH et al., 1988). LANGLANDS et al. (1981) sugerem que os TMs alteram profundamente $\mathrm{o}$ equilíbrio entre $\mathrm{Cu}$ livre e cuproproteínas, inclusive comprometendo a atividade enzimática. MASON (1990) tem sugerido que o complexo $\mathrm{Cu}$ :TM:albumina formado pode ser hidrolizado e seus componentes eliminados pela urina. Os TMs podem diminuir as reservas corporais de $\mathrm{Cu}$ por interferência com a circulação entero-hepática via secreção biliar de $\mathrm{Cu}$ (HUMPHRIES et al., 1986).

LANGLANDS et al. (1981) têm sugerido que o incremento inicial no plasma de $\mathrm{Cu}$ insolúvel em TCA, após a administração parenteral de TMs, representa o $\mathrm{Cu}$ mobilizado do fígado, uma vez que as reservas do fígado diminuem progressivamente. Estudos demonstram que o $\mathrm{TM}_{4}$ intravenoso afasta o $\mathrm{Cu}$ tanto dos lisossomas como do citosol dos hepatócitos; esses efeitos provavelmente explicam o incremento na secreção biliar de $\mathrm{Cu}$, observado em ovelhas tratadas com $\mathrm{TM}_{4}$. $\mathrm{O}$ alto conteúdo de carboidratos fermentáveis dos alimentos digeríveis pode diminuir o $\mathrm{pH}$ e aumentar a disponibilidade de $\mathrm{Cu}$ por incremento da absorção de $\mathrm{S}^{2-}$ e decomposição química dos TMs; isto pode explicar porque o Mo (2,5 - 5,0mg/kg MS) não acelera o índice de depleção do $\mathrm{Cu}$ hepático em ovelhas alimentadas com uma dieta de grãos.

Sabe-se que os ovinos são sensíveis à intoxicação por $\mathrm{Cu}$ e que a administração de tetratiomolibdato de amônia $\left(\mathrm{TM}_{4}-\mathrm{NH} 3\right)$ reduz a severidade desta condição (HUMPHRIES et al., 1986). SUTTLE (1991) reportou que bezerros que receberam quantidades excessivas de $\mathrm{Cu}$, no sucedâneo do leite (10mg de $\mathrm{Cu} / \mathrm{kg}$ de $\mathrm{MS}$ ), desenvolveram sintomas de cupro toxicidades similares àqueles observados em ovinos, porém, após a aplicação de $\mathrm{TM}_{4}-$ $\mathrm{NH} 3$, os sintomas desapareceram. O maior problema do uso de $\mathrm{TM}_{4}-\mathrm{NH} 3$ está na retenção sistemática de Mo devido ao fato de não não todo o $\mathrm{TM}_{4}-\mathrm{NH} 3$ ser excretado (MASON et al., 1988). Ele é retido em alguns órgãos, principalmente cérebro e pituitária, que se constituem nos órgãos alvo (HAYWOOD, $\boldsymbol{e} t$ al., 1998). O Glicinato de cobre produz maior aumento da atividade sérica da ceruloplasmina e ganho de peso, podendo ser considerado de utilidade no tratamento de estados de hipocuprose produzidos pelo excesso de Mo e $\mathrm{SO}_{4}$ (QUIROGA, et al., 1998a; QUIROGA, et al., 1998b).

Bovinos suplementados com Mo e S tiveram níveis baixos de cobre comparados com os animais não suplementados (CERONE, et al., 1998). Nesta pesquisa, também se observou que o número e porcentagem de neutrófilos não foram alterados, porém a concentração intracelular de $\mathrm{Cu}$ nos neutrófilos, diminuiu no grupo suplementado com molibdênio e enxofre. Os caprinos podem apresentar características mais similares aos ovinos que aos bovinos, referente à resposta adversa para elevadas quantidades de $\mathrm{Cu}$ dietético, contudo informações sobre a interação Cu-Mo-S são limitadas nesta espécie (GALBRAITH et al., 1997).

Estudos de permeabilidade placentária demonstraram que o Mo atravessa a placenta, porém parece altamente improvável que forme complexos TMs-proteína ou se acumulem causando efeitos prejudiciais ao metabolismo placentário. As barreiras fisiológicas podem, proteger parcialmente o feto e órgãos como cérebro e medula espinhal, dos efeitos prejudiciais dos TMs circulantes (SUTTLE, 1991).

Injeções repetidas de $\mathrm{TM}_{4}$ em carneiros reduziram as ondulações e resistência da lã, efeitos similares aos observados na deficiência de $\mathrm{Cu}$, sendo 
que as concentrações de $\mathrm{Cu}$ no fígado e sangue encontraram-se normais. Uma possível explicação é que se produz acumulação de TMs na pele e diminuição local de $\mathrm{Cu}$, afetando o desenvolvimento de pêlos e da lã (GOONERATNE $\boldsymbol{e}$ t al., 1989). O tecido conjuntivo pode ser outro local vulnerável, dadas as mudanças no periósteo e tecido cartilaginoso de cordeiros expostos ao Mo (MASON, 1990).

Durante vários anos, a suplementação de cobre estava limitada a formas inorgânicas do elemento, mas hoje as formas quelatadas têm começado a ser mais utilizadas pela maior biodisponibilidade destas fontes (HEMKEN, et al., 1995). WARD et al. (1993), reportaram que na presença de níveis altos de Mo, o proteinado de $\mathrm{Cu}$ manteve mais estável a concentração plasmática de $\mathrm{Cu}$ em bovinos e teve maior biodisponibilidade do elemento, quando compararam com o grupo suplementado com sulfato de cobre $\left(\mathrm{CuSO}_{4}\right)$. É relatado que durante 73 dias de estudo com ovelhas não existiu toxicidade do cobre $(30 \mathrm{mg} / \mathrm{kg}$ ) quando usado na forma de $\mathrm{CuSO}_{4}$ ou proteinado (Eckert, et al., 1999). No mesmo estudo, os autores também concluíram que os animais suplementados com proteinado tiveram maior atividade ceruplasmina e menor nível de $\mathrm{Cu}$ no fígado quando comparado com as ovelhas suplementadas com $\mathrm{CuSO}_{4}$.

\section{INTERAÇÃO CU-MO-S}

MILTIMORE \& MASON (1971) argumentaram que mais importante que a ingestão absoluta de $\mathrm{Cu}$ e/ou Mo seria a concentração relativa desses elementos nas pastagens. Por exemplo, no Reino Unido, a "peat scours" era comum nos animais mantidos em pastagens com concentrações subnormais de $\mathrm{Cu}$ e conteúdo maior do que o normal de Mo. No mesmo país, a "teart syndrome" ocorria em pastagens cujo conteúdo de $\mathrm{Cu}$ mostrava-se aparentemente normal, mas a concentração de Mo era bem maior que a encontrada nas pastagens mencionadas anteriormente. Baseados nisso, ELGALLAD et al. (1983) sugeriram que uma relação $\mathrm{Cu}: \mathrm{Mo}$, na dieta menor que 2 , pode provocar deficiência de $\mathrm{Cu}$ em bovinos, ressaltando que é mais importante a relação $\mathrm{Cu}: \mathrm{Mo}$ que os níveis dietéticos dos elementos. Concentrações de Mo entre cinco e 6ppm podem causar intoxicação em vacas se a relação $\mathrm{Cu}$ :Mo for menor que 2:1 e se níveis altos de sulfato estão presentes na dieta; porém concentrações de Mo menores que $1 \mathrm{ppm}$ podem provocar efeitos tóxicos, se a entrada de $\mathrm{Cu}$ é lenta e a entrada de enxofre é alta (WITTENBERG \& BOILA, 1988). Há outra teoria sobre a possibilidade do Mo ser mais importante nessa interação que o $\mathrm{Cu}$, mostrando efeitos tóxicos no metabolismo que o $\mathrm{Cu}$ pode anu- lar (HUMPHRIES et $\boldsymbol{a l}$., 1983; LADEFOGED \& STÜRUP, 1995), sendo, portanto um problema de excesso de Mo antes que deficiência de $\mathrm{Cu}$, em outras palavras molibdenosis e não hipocuprosis.

Sabe-se que, na deficiência de $\mathrm{Cu}$, diminui a atividade da superoxido dismutase (XIN et al., 1991), que é uma cupro-enzima que catalisa a dismutação de $\mathrm{O}_{2}{ }^{-}$para $\mathrm{H}_{2} \mathrm{O}_{2}$, que é o grupo oxidante que participa nas reações de defesa dos neutrófilos (BABIOR, et al., 1973). A menor formação de $\mathrm{H}_{2} \mathrm{O}_{2}$ em relação a $\mathrm{O}_{2}{ }^{-}$pode causar peroxidação dos lípides da membrana celular alterando sua estrutura (WOLFF, et al., 1986). No caso da alteração dos componentes da membrana dos leucócitos, inicia-se o processo de síntese de prostaglandinas e leukotrienos (CLARK et al., 1995) danificando a estrutura do receptor, prejudicando a função fagocitária dos macrófagos (LOEGERING, et al., 1996), como conseqüência, os animais estarão expostos a infecções severas, por queda da função das células fagocitárias, comprometendo o sistema imune de defesa não específica (WARD, et al. 1997). O efeito da deficiência de $\mathrm{Cu}$ por baixa ingestão ou por influencia do nível alto de Mo produz resultados inconsistentes na função imune, já que outras pesquisas, indicaram que esta deficiência não poderia alterar a resposta imune em bovinos (BREM, et al., 1999; FAILLA \& HOPKINS, 1998; WARD, et al., 1997).

\section{FATORES QUE INFLUENCIAM INTERAÇÃO CU-MO-S}

Alguns agentes que competem com o molibdênio pelo sulfato no rúmen influenciam, provavelmente, o curso da interação $\mathrm{Cu}$ - Mo - S. O ferro é potencialmente o exemplo mais importante para animais em pastagem, porque grandes quantidades de Fe são ingeridas através dos solos e provavelmente uma fração se ligue ao sulfato. A inibição do metabolismo do $\mathrm{Cu}$ por suplementos de $\mathrm{Fe}$ em ovelhas tem sido atribuída particularmente à captura de $\mathrm{S}_{2}{ }^{2-}$ como $\mathrm{FeS}$ no rúmen, seguida pela liberação de $\mathrm{S}_{2}$ no meio ácido do abomaso para formar $\mathrm{CuS}$ (HUMPHRIES $\boldsymbol{e t}$ al., 1983; SUTTLE $\boldsymbol{e t}$ al., 1984). Outros metais que formam sulfatos ácidos lábeis podem ter efeitos similares àqueles exibidos pelo $\mathrm{Fe}$ (exemplo o Mn), mas aqueles que formam sulfatos não solúveis em ácidos (chumbo e cádmio) podem ser protegidos por restrição na formação tanto de TMs como CuS (SUTTLE et al., 1984).

Parece que a forma do Mo (molibdato de sódio, molibdato de amônia ou trioxido de molibdênio) não influencia na biodisponibilidade deste elemento, já que as concentrações de Mo no músculo e soro em ovelhas foram similares usando estas três 
fontes de molibdênio (POTT $\boldsymbol{e t} \boldsymbol{a l}$., 1999b). Nesta pesquisa, também foi observado que o aumento do Mo da dieta incrementou a concentração de $\mathrm{Cu}$ nos rins, o nível serico total de $\mathrm{Cu}$ e $\mathrm{Cu}$ insolúvel em ATC e excreção diária de cobre pelas fezes. Aparentemente, a biodisponibilidade do Mo estaria dependendo mais da concentração do elemento na dieta que do tempo de consumo, visto que POTT et al. (1999a) não observaram diferença quando os animais foram expostos a 14 ou 28 dias de ingestão de Mo, mas quando comparados quatro níveis de Mo na dieta $(0,15,30$ e 45ppm) mostraram que a excreção urinária e fecal de Mo aumentou em forma linear.

\section{CONCLUSÕES}

A interação $\mathrm{Cu}-\mathrm{Mo}-\mathrm{S}$ pode ser desenvolvida no trato digestivo ou ocorre durante a metabolização, devido à redução de sulfato para sulfeto no rúmen; logo este sulfeto reage com Mo para formar TMs; e posteriormente a formação de $\mathrm{Cu}-\mathrm{TMs}$ (CuMoS4) que são altamente insolúveis e não utilizáveis. Então a interação manifesta-se com maior intensidade em ruminantes devido à relação do enxofre com o metabolismo rumenal.

O Molibdênio, especialmente quando o enxofre está presente, diminui o depósito de cobre nos órgãos e a síntese de ceruloplasmina, decorrendo a queda na excreção de $\mathrm{Cu}$ pela bílis e o aumento na excreção urinária. $\mathrm{O}$ incremento do $\mathrm{Cu}$ dietético reduz os depósitos de Mo no fígado.Quando o nível de S aumenta, a excreção urinária de Mo eleva-se substancialmente, enquanto o depósito nos tecidos diminui proporcionalmente. A mobilização do $\mathrm{Cu}$ hepático, produzido pela presença de Mo, é utilizado como fundamento no tratamento de cuprosis. Os efeitos da interação dependem da relação $\mathrm{Cu}: \mathrm{Mo}$, sendo secundárias as concentrações excessivas de $\mathrm{Cu}$ ou Mo na dieta isoladamente.

\section{REFERÊNCIAS BIBLIOGRÁFICAS}

BABIOR, B.M., KIPNES, R.S., CURNUTTE, J.T. Biological defense mechanisms. The production by leukocytes of superoxide, a potencial bacterial agent. Journal Clinical Investigation, v.52, p.741-744, 1973.

BREM, J.J., PERIOLO, O. H., ROUX, J.P. Linfoictos "T" y respuesta humoral antiaftosa em bovinos com diferente disponibilidad de cobre y molibdemo. Revista de Medicina Veterinaria, v.80, p.14-18, 1999.

CARDOSO, E.C., McDOWELL, L.R., VALE, W.G., et al. Copper and molybdenum status of cattle and buffaloes in Marajo island, Brazil. International Journal Animal Science, v.12, p.57-60, 1997.

CERONE, S., SANSINANEA, A., STREITENBERGER, S., et al. Bovine neutrophil functionality in molybdenum-induced copper deficiency. Nutrition Research, v.18, p.557-566, 1998

ClARK, C.K., ANSOTEGUi, R.P., PATERSON, J.A. Mineral nutrition of the beef cow to impact immunologic resposnse. The Bovine Practitioner, v.29, p.30-37, 1995.

DICK, A.T., DEWEY, D.W., GAWTHORNE, J.M. Thiomolybdates and the copper - molybdenum - sulphur interaction in ruminant nutrition. Journal Agricultural Science, v.85, p.567-568, 1975.

ECKERT, G.E., GREENE, L.W., CARSTENS, G.E., $\boldsymbol{e} \boldsymbol{t}$ al Copper status of ewes fed increasing amounts of copper from copper sulfate or copper proteinate. Journal Animal Science, v.77, p.244-249, 1999

El-GALLAD, T.T., MILLS, C.F., BREMNER, I., et $\boldsymbol{a l}$. Thiomolybdates in rumen contents and rumen cultures. Journal Inorganic Biology, v.18, p.323 -334, 1983.

FAILLA, M.L., HOPKINS, R.G. Is low copper status immunosuppressive?. Nutrition Reviews, v.56, p.S59-S64, 1998.

GALBRAITH H., CHIGWADA, W, SCAIFE, J. R., et $\boldsymbol{a l}$. The effect of dietary molybdenum supplementation on tissue copper concentrations, mohair fibre and carcass characteristics of growing Angora goats. Animal Feed Science Tecnology, v.67, p.83 - 90, 1997.

GOONERATNE, S.R., BUCKLEY, W.T., CHRISTENSEN, D.A. Rewiew of copper deficiency and metabolism in ruminats Canadian Journal Animal Science, v.69, p.819-845, 1989.

HAYWOOD, S., DINCER, Z., HOLDING, J., et al. Metal (molybdenum, copper) acumulation and retention in brain, pituitary and other organs of ammonium tetrathiomolybdatetreated sheep. British Journal Nutrition, v.79, p.329-331, 1998.

HEMKEN, R.W., CLARK, T.W., DU, Z. Copper: Its role in animal nutrition. In: Biotechnology in the feed industry: Proceedings of Alltech's 11th Annual Symposium, Lyons P, Jaques K (eds). Nottinghan University Press, Nottinghan p.35-39, 1995.

HUMPHRIES, W.R., PHILLIPPO, M., YOUNG, B.W., et al. The influence of dietary iron and molybdenum on copper metabolism in calves. British Journal Nutrition, v.49, p.7786,1983

HUMPHRIES, W.R., MILLS, C.F., GREIG, A., et al. Use of ammonium tetrathiomolybdate in the treatment of copper poisoning in sheep. Veterinary Record, v.119, p.596 - 598, 1986.

LADEFOGED, O. STÜRUP, S. Copper deficiency in cattle, sheep and horses caused by excess molybdenum from fly ash: A case report. Veterinary and Human Toxicology, v.37, p.63-65, 1995 .

LANGLANDS, J.P., BOWLES, J.E. DONALD, G.E., $\boldsymbol{e} \boldsymbol{t} \boldsymbol{a l}$. Copper status of sheep grazing pastures fertilized with sulfur and molybdenum. Australian Journal Agriculture Research, v.32, p.479-486, 1981.

LOERING, D.J., RALEY, M.J., REHO, T.A., et al., Macrophage dysfunction following the phagocytosis of IgG-coated erythrocytes: production of lipid peroxidation products. Journal Leukocyte Biology, v.59, p.357-362, 1996. 
MASON, J. The biochemical pathogenesis of molybdenuminduced copper deficiency syndromes in ruminants: Towards the final chapter. Irish Veterinary Journal, v.43, p.18 - 21, 1990.

MASON, J., KELLEHER, C. A., LETTERS J. The demonstration of protein-bound $99 \mathrm{Mo}-\mathrm{di}-$ and trithiomolybdate in sheep plasma after the infusion of 99 Mo-labelled molybdate into the rumen. British Journal Nutrition, v.48, p.391 - 397, 1982.

MASON, J., LAMAND, M., TRESSOL, J.C., et al. Studies on the changes in systemic copper metabolism and excretion produced by the intravenous administration of trithiomolybdate in sheep. British Journal Nutrition, v.59, p.289-300. 1988

McDOWELL, L.R., CONRAD, J.H., ELLIS, G.L., $\boldsymbol{e}$ t al Nutrición mineral para rumiantes bajo pastoreo en los trópicos. Asociación latinoamericana de Producción Animal (ALPA), v.23, p.25-39, 1988.

MILTIMORE, J.E., MASON, J.L. Cooper to molybdenun ratio and molybdenun and copper concentration in ruminan feeds. Canadian Journal Animal Science, v.51, p.193 - 200, 1971.

MORAES, S.S., SILVA, G.N., DOBEREINER, J. Microelementos minerais e a "cara inchada" dos bovinos. Pesquisa Veterinária Brasileira, v.14, p.25-33, 1994.

POTT, E.B., ALMEIDA, I.L., BRUM, P.A.R., et al. Nutrição mineral de bovinos de corte no Pantanal Mato-grossense. 2. Micronutrientes na Nhecolândia (parte central). Pesquisa Agropecuaria Brasileira, v.24, p.109-126, 1989.

POTT, E.B., HENRY, P.R., RAO, P.V., et al. Estimated relative bioavailability of supplemental inorganic molybdenum sources and their effect on tissue molybdenum and copper concentrations in lambs. Animal Feed Science and Technology, v.79, p.107-117, 1999a.

POTT, E.B., HENRY, P.R., ZANETTI, M.A., et al. Effects of high dietary molybdenum concentration and duration of feeding time on molybdenum and copper metabolism in sheep. Animal Feed Science and Technology, v.79, p.93$105,1999 b$.

PRICE, J., WILL, A.M., PASCHALERIS, G., et al. Identification of thiomolybdates in digesta and plasma from sheep after administration of Mo-labelled compounds into the rumen. British Journal Nutrition, v.58, p.127 - 138, 1987.

QUIROGA, M.A., IGARZA, L.M., LANDA, R.D. et al Evaluación farmacotécnica, toxicologica y terapeutica del
Glicinato de Cobre en bovinos con Molibdenosis. 1o Parte. Revista de Medicina Veterinaria, v.79, p.311-316, 1998a.

QUIROGA, M.A., IGAZA, L.M., LANDA, R.D. $\boldsymbol{e t}$ al. Evaluación farmacotécnica, toxicologica y terapeutica del Glicinato de Cobre en bovinos con Molibdenosis. 2o Parte. Revista de Medicina Veterinaria, v.79, p.357-364, 1998b.

SMITH, E.L., HIL, R.L., LEHMAN, I.R., et. al. Bioquímica dos mamíferos. Rio de Janeiro: $7^{\circ}$ ed. Guanabara Koogan. 1988. $620 \mathrm{p}$.

SUTTLE, N.F. Recents studies of the copper-molybdenum antagonist. Proceeding Nutrition Society, v.33, p.299-305, 1974

SUTTLE, N.F. The interactions between copper, molybdenum, and sulphur in ruminant nutrition. Annual Veterinary Nutrition, v.11, p.121-140, 1991.

SUTTLE, N.F., ABRAHAMS, P., HORNTON, I. The role of a soil $\mathrm{x}$ dietary sulphur interaction in the impairment of copper absortion by ingested soil in sheep. Journal Agriculture Science (Cambridge), v.103, p.81-86, 1984.

TOKARNIA, C.H., DÓBEREINER, J., MORAES, S.S., et al Deficiências e desequilíbrios minerais em bovinos e ovinosrevisão dos estudos realizados no Brasil de 1987-1998. Pesquisa Veterinária Brasileira, v.19, p.47-62, 1999.

WARD, J.D., GENGELBACH, G.P., SPEARS, J.W. The effects of copper deficiency with or without high dietary iron or molybdenum on immune function of cattle. Journal Animal Science, v.75, p.1400-1408. 1997.

WARD, J.D., SPEARS, J.W., KEGLEY, E.B. Effect of copper level and source (copper lysine vs copper sulfate) on copper status, performance, and immune resposnse in growing steers fed diets with or without supplemental molybdenum and sulfur. Journal Animal Science, v.71, p.2748-2754, 1993.

WITTENBERG, K.M., BOILA, R.J. Supplementary copper for growing cattle consuming diets high in molybdenum or molybdenum plus sulfur. Canadian Journal Animal Science, v.68, p.1143-1154, 1988.

WOLFF, S.P., GARNER, A., DEAN, R.T. Free radicals, lipids and protein degradation. Trends Biochemical Science, v.11, p.27-31, 1986.

XIN, Z., WATERMAN, D.F., HEMKEN, R.W. et al. Effects of copper status on neutrophil function, superoxide dismutase, and copper distribution in steers. Journal Dairy Science, v.74, p.3078-3085, 1991.

Ciência Rural, v. 31, n. 6, 2001. 